\title{
Critical temperature of Ising films with cubic lattices
}

\author{
D. L. Lin \\ Department of Physics, Hong Kong University of Science and Technology, Clear Water Bay, Kowloon, Hong Kong \\ and Department of Physics and Astronomy, State University of New York at Buffalo, Buffalo, New York 14260-1500* \\ Hao $\mathrm{Che}^{\dagger}$ and Wei Lai \\ Department of Physics and Astronomy, State University of New York at Buffalo, Buffalo, New York 14260-1500 \\ Thomas F. George \\ Department of Physics and Department of Chemistry, Washington State University, Pullman, Washington 99164-1046
}

(Received 29 June 1993)

\begin{abstract}
Following the approach recently proposed by Lin, Che, and Xia [Phys. Rev. A 46, 1805 (1992)], we calculate the critical temperature $T_{c}$ for Ising films with lattice structures other than simple cubic by means of the variational cumulant expansion (VCE) of the free energy of the spin system. The critical temperature is derived in terms of the ratio of two successive cumulants to an arbitrary order of accuracy. Since the cumulant of any order $m$ can be calculated analytically by a graphic method, analytical expressions of $T_{c}$ can be derived, in principle, as functions of the number $L$ of spin layers in the film to an arbitrary order of accuracy in the VCE. We describe the graphic method in great detail in this paper, and calculate $T_{c}$ up to the fourth-order cumulant for films of body-centered-cubic lattice and to the third order for films of face-centered-cubic lattice. Our results show that in the limit of $L \rightarrow \infty, T_{c}$ approaches the corresponding bulk value rather quickly with increasing $m$ in both cases.
\end{abstract}

PACS number(s): 05.70.Jk, 75.10.Hk, 75.40.Cx, 75.70.Ak

\section{INTRODUCTION}

Recently, a completely different to the calculation of the critical temperature $T_{c}$ of Ising films has been proposed [1] on the basis of the variational cumulant expansion (VCE). The VCE developed in recent years for discussions of lattice gauge field models [2-5] has been extended to treat the Ising model in statistical physics $[6,7]$. By expanding the free energy in the VCE to an arbitrary order of accuracy, it is shown in Ref. [1] (to be referred to as I) that $T_{c}$ is expressed in a limiting form in terms of the ratio of two successive cumulants which are constructed analytically by means of recursion relations derived in Ref. [7]. The formalism is so general that it is valid for any spin, and any lattice structure. The geometry of the crystal does not have to be specified either. For a uniform spin- $\frac{1}{2}$ hypercubic film of $(d+1)$ dimension, $T_{c}$ is calculated analytically in $I$, up to the fourth-order cumulant, as a function of $d$ and $L$, where $L$ stands for the number of spin layers in the hyperfilm.

The procedure of calculation consists of two stages. In the first, we derive the cumulants from recursion relations. The lattice structure and crystal geometry are not specified and therefore the results are valid in general. The lattice and geometry are specified in the second stage

\footnotetext{
*Permanent address.

†Present address: Department of Physics, University of Texas at Arlington, Arlington, TX 76019.
}

and topologically equivalent terms are summed up by means of the graph technique. Since many of the complications are already involved in the first stage, the amount of work involved in the second is greatly reduced. For example, there are only six distinct connected diagrams involved in our calculation of the fourth-order cumulants for the hypercubic Ising film in [1].

It is found that to the first-order correction, our result is identical to that of the mean-field theory. This is easily understood because all the bonds are decoupled in the first-order cumulant. Bethe's approximation is found to be exactly the same as our third-order results only for $L=1,2$, and $\infty$, for which Bethe's assumption of the mean coordination number $[6(L-2)+10] / L$ is exact. We believe that by adding more terms in the expansion, our results are expected to quickly approach those of the high-temperature series expansion extrapolation (HTSEE) obtained up to twelfth order in the expansion series for films up to seven spin layers [8]. Computer codes with the help of softwares such as Schoonschip [9] may be developed to calculate $T_{c}$ with much higher accuracy.

Due to the rapid development of technology, a great deal of effort has been devoted to experimental measurements of the critical temperature for films of Ising-like materials with various lattice structures [10-15]. It is therefore interesting to calculate $T_{c}$ of Ising films with different lattice structures. We present in this paper our theoretical results for films with body-centered-cubic (bcc) and face-centered-cubic (fcc) lattices. Again, analytic expressions are given for $T_{c}$ as functions of the number of spin layers in the film and numerical results are plot- 
ted. The corresponding bulk $T_{c}$ values obtained from the HTSEE [16] are also given for comparison. It is observed that the first few terms of VCE results converge quickly toward these values. A more complete calculation including higher-order terms is expected to yield rather accurate critical temperature in the limit of $L \rightarrow \infty$.

\section{REVIEW OF THE THEORY}

We consider a spin system with the Hamiltonian

$$
H=\frac{1}{s^{2}} \sum_{\langle i, j\rangle} J_{i j} s_{i}^{z} s_{j}^{z}-\frac{\mu}{s} \sum_{i} H_{i} s_{i}^{z},
$$

where $s^{z}=-s,-s+1, \ldots, s, J_{i j}$ stands for the exchange energy between the spin pair $i$ and $j, \mu$ represents the magnetic moment of a spin, and $H_{i}$ is the inhomogeneous external field at the lattice site $i$ in the $z$ direction. The sum is taken over every pair only once. The action $S$ for the system is defined as

$S=-\frac{1}{s^{2}} \sum_{\langle i, j\rangle} \frac{J_{i j}}{k_{B} T} s_{i}^{z} s_{j}^{z}-\frac{1}{s} \frac{\mu}{k_{B} T} \sum_{i} H_{i} s_{i}^{z}=\frac{1}{k_{B} T} X$,

and the corresponding trial action $S_{0}$ for the noninteracting system is

$$
S_{0}=\frac{1}{s} \sum_{i}\left[\xi_{i}+\frac{\mu H_{i}}{k_{B} T}\right] s_{i}^{z} .
$$

Here $\xi_{i}$ have been introduced as variation parameters which, as we shall see later, serve as the order parameters of our system. The VCE of the free energy up to the order $m$ is given by

$$
W \approx W_{0}-\sum_{n=1}^{m} \frac{1}{n !}\left\langle\left(S-S_{0}\right)^{n}\right\rangle_{c}=W_{\mathrm{eff}, m},
$$

where $W_{0}$ represents the free energy of the corresponding noninteracting system and the symbol \langle\rangle$_{c}$ denotes the cumulant average of the enclosed quantity over the Boltzmann weight $e^{S_{0}}$.

For a uniform spin- $\frac{1}{2}$ Ising film in the absence of external fields, we have $J_{i j}=J, \xi_{i}=\xi, s^{z}= \pm \frac{1}{2}$, and $H_{i}=0$. From the analytical properties of the free energy $W_{\text {eff, } 1}$ corrected to the first order, a conjecture has been proposed in [1] that the critical temperature $T_{c}$ can be determined to an arbitrary order $m$ of accuracy by locating the bifurcation point of the $m$ th-order VCE of the free energy, namely, $W_{\text {eff, } m}$. In other words, $T_{c}$ to the $m$ th-order cumulant approximation is determined by the condition

$$
\left.\frac{\delta^{2}}{\delta \xi^{2}} \boldsymbol{W}_{\mathrm{eff}, m}\left(\boldsymbol{\Theta}_{c}, \xi\right)\right|_{\xi=0}=0
$$

where we have defined the reduced temperature $\Theta_{c}=k_{B} T_{c} / J$. As has been discussed in $\mathrm{I}$, starting from the first-order cumulant and making use of recursion relations for the cumulants, one can prove by mathematical induction that Eq. (5) reduces to

$$
\left[\frac{\delta^{2}}{\delta \xi^{2}}\left\langle S^{m-1}\right\rangle_{c}-\frac{1}{r_{i}} \frac{\delta^{2}}{\delta \xi^{2}}\left\langle S^{m}\right\rangle_{c}\right]_{\xi=0}=0
$$

up to any order $m$. Equation (6) implies immediately that $T_{c}^{(m)}$ calculated to the $m$ th order in the VCE is given by

$$
k_{B} T_{c}^{(m)}=\frac{1}{m} \frac{\delta^{2}}{\delta \xi^{2}}\left\langle X^{m}\right\rangle_{c} /\left.\frac{\delta^{2}}{\delta \xi^{2}}\left\langle X^{m-1}\right\rangle_{c}\right|_{\xi=0}
$$

for $m \geq 2$, where $X=k_{B} T S$ as is defined in Eq. (2) and the energy is expressed in the unit of $J$. Hence the critical temperature for the system is obtained simply by extending $m$ to infinity, or

$$
T_{c}=\lim _{m \rightarrow \infty} T_{c}^{(m)} \text {. }
$$

\section{METHOD OF CALCULATION}

The cumulant $\left\langle X^{m}\right\rangle_{c}$ can be calculated order by order from the recursion formula [1]

$$
\begin{aligned}
\left\langle S^{p+1}\right\rangle_{0}=\sum_{\langle i, j\rangle} \beta_{i j}[ & \frac{\delta^{2}}{\delta \xi_{i} \delta \xi_{j}}+2 \frac{\delta \ln Z_{0}}{\delta \xi_{i}} \frac{\delta}{\delta \xi_{j}} \\
& \left.+\frac{\delta \ln Z_{0}}{\delta \xi_{i}} \frac{\delta \ln Z_{0}}{\delta \xi_{j}}+\frac{\delta^{2} \ln Z_{0}}{\delta \xi_{i} \delta \xi_{j}}\right]\left\langle S^{p}\right\rangle_{0},
\end{aligned}
$$

where $p$ is an integer and $\beta_{i j}$ measures $J_{i j}$ in the unit of $k_{B} T$. It is useful to point out that Eq. (9) is still valid in the presence of a nonzero external field provided that $\xi_{i}$ is replaced by $x_{i}=\xi_{i}+\mu H_{i} / k_{B} T$. For a system of $N$ spins, the moments are polynomials of $N$ and are related to cumulants of the same order by the well-known relation.

$$
\left\langle S^{p}\right\rangle_{c}=\left(\text { the linear term of }\left\langle S^{p}\right\rangle_{0}\right) .
$$

In other words, we can extract the cumulant $\left\langle S^{p}\right\rangle_{c}$ and hence $\left\langle X^{p}\right\rangle_{c}$ out from the moment $\left\langle S^{p}\right\rangle_{0}$ simply by taking the term linear in $N$.

At this point, it may be in order to remark that the cumulants $\left\langle S^{p}\right\rangle_{c}$ found in Eq. (10) are very general in the sense that the lattice structure and the geometry of the system are not specified. For example, the lowest two orders of cumulants for the spin- $\frac{1}{2}$ Ising model with nearest-neighbor interactions are given by

$$
\begin{aligned}
\left\langle S^{1}\right\rangle_{c}=\frac{1}{2} \sum_{i} \beta_{i, i+\mu} y_{i} y_{i+\mu} & \\
\left\langle S^{2}\right\rangle_{c}=\frac{1}{2} \sum_{i}[ & \beta_{i, i+\mu} \beta_{i, i+\mu}\left(1-y_{i}^{2}\right)\left(1-y_{i+\mu}^{2}\right) \\
& \left.\quad+2 \beta_{i, i+\mu} \beta_{i, i+\nu} y_{i+\mu} y_{i+v}\left(1-y_{i}^{2}\right)\right]
\end{aligned}
$$

where we have defined

$$
y_{i}=\tanh \xi_{i}
$$

and the subscripts $i+\mu$ and $i+v$ run over all the nearest neighbors of the lattice site $i$. Explicit expressions for higher-order terms are very long and it is not instructive to write them down completely here. Because all quantities on the right-hand sides of Eqs. (11) and (12) depend explicitly on lattice sites, these equations are generally good for the calculation of cumulants for inhomogeneous 
Ising systems of various lattice structures with different geometry.

We are now in the position to sum over the equivalent terms in Eqs. (11) and (12) for uniform Ising films of $N_{1}$ spin layers. We notice that $\xi_{i}=\xi$ and $\beta_{i, i+\mu}=\beta$ with positive integer $i$ for a uniform spin system. Therefore, Eqs. (11) and (12) can be written as

$$
\begin{aligned}
\left\langle S^{1}\right\rangle_{c} & =\frac{1}{2} \beta N_{2}[l, l+\mu] y^{2}, \\
\left\langle S^{2}\right\rangle_{c}=\frac{1}{2} \beta^{2} N_{2}\left\{[l, l+\mu]\left(1-y^{2}\right)^{2}\right. & \\
& \left.+2[l, l+\mu, l+v] y^{2}\left(1-y^{2}\right)\right\},
\end{aligned}
$$

where $N_{2}$ represents the number of lattice sites in each spin layer and tends to infinity in our present cases. This infinity does not appear in $T_{c}$ because of cancellation in $X^{m}$ and $X^{m-1}$ according to Eq. (7). The symbol $[l, l+\mu]$ stands for the number of graphs in which two nearestneighboring sites are connected by a bond, and these connected graphs are embedded on the lattice site $l$. Figure 1(a) shows the type of graph we have to sum in evaluating $\left\langle S^{1}\right\rangle_{c}$. The symbol $[l, l+\mu, l+v]$ is the number of graphs of the type shown in Fig. 1(b). Both bonds must be summed independently over all the nearest neighboring sites of $l$. It is noted that the lattice site $l$ itself has to be summed over all sites in the $z$ direction. Thus, there are only two types of graphs to be summed up to the second-order cumulant.

It is now a simple matter to calculate the critical temperature to the second-order cumulant. Inserting the cumulants found from Eqs. (14) and (15) into Eq. (7), and remembering the relation $X=k_{B} T S$, we find

$$
k_{B} T_{c}^{(2)} / J=\frac{[l, l+\mu, l+v]-[l, l+\mu]}{[l, l+\mu]} .
$$

At this point, it is important to remark that strictly speaking, Eq. (7) holds true only for $m \geq 2$.

For $m=1$, Eq. (6) implies that (a)

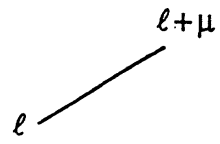

(b)

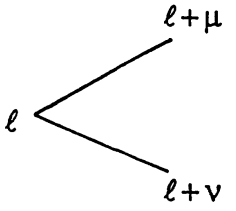

FIG. 1. Graphs contributing to the first- and second-order cumulants $\left\langle S^{1}\right\rangle_{c}$ and $\left\langle S^{2}\right\rangle_{c}$.

$$
k_{B} T_{c}^{(1)}=\frac{\delta^{2}}{\delta \xi^{2}}\left\langle X^{1}\right\rangle_{c} /\left.\frac{\delta^{2}}{\delta \xi^{2}}\left\langle S_{0}\right\rangle_{c}\right|_{\xi=0},
$$

where we have defined

$$
\left\langle S_{0}\right\rangle_{c}=\sum_{i} \xi_{i} y_{i}
$$

As has been shown in I, Eq. (17) yields the mean-field results. With the help of Eqs. (14) and (18), we immediately find from (17) that

$$
k_{B} T_{c}^{(1)} / J=[l, l+\mu] /[l],
$$

where the symbol $[l]$ is simply a sum of "points." In other words, $[l]=N_{1}$ which represents the number of lattice points in the $z$ direction, or the number of spin layers $L=N_{1}$.

Equations (16) and (19) are valid for both bcc and fcc lattices as well as for the simple cubic (sc) lattice. This is, however, not true in general for higher-order results. As a matter of fact, an explicit calculation up to the thirdorder cumulants yields

$$
k_{B} T_{c}^{(3)} / J=\frac{2[l, l+\mu]-6[l, l+\mu, l+v]+3[l, l+\mu, l+v+\eta]}{3\{-[l, l+\mu]+[l, l+\mu, l+v]\}},
$$

for bcc lattices, and

$$
k_{B} T_{c}^{(3)} / J=\frac{2[l, l+\mu]-6[l, l+\mu, l+v]+3[l, l+\mu, l+v+\eta]-3[l, l+\mu+v=l+\eta]}{3\{-[l, l+\mu]+[l, l+\mu, l+v]\}}
$$

for fcc lattices. The extra term appears in Eq. (21) because three connected bonds can form a closed loop in fcc lattices while at least four bonds are needed to form a loop in bcc lattices. The symbols $[l, l+\mu+v],[l, l+\mu, l+v+\eta]$, and $[l, l+\mu+v=l+\eta]$ are, respectively, the numbers of graphs of the type (a), (b), and (c) illustrated in Fig. 2. We note, however, that Figs. 1(b) and 2(a) are topologically equivalent.

In Eq. (21), the term corresponding to a closed loop represents the contribution from the self-correlation of the initial spin at $l$, and is expected to be very important as compared to other graphs of the same order. From our experience with the simple-cubic lattice in I, the first loop graph appears in the fourth order. There we have found that the change of convergence rate increases significantly in the fourth order. In other words, the fourth-order correction improves the resulting $T_{c}$ more significantly than the third-order correction for Ising films of simple-cubic lattice structure. For this reason, we also calculate $T_{c}$ up to the lowest order in which a closed loop appears in the graph. In the present paper, we therefore calculate $T_{c}$ up to the fourth-order cumulant for a bcc Ising film, and only to third order for a fcc Ising film. To fourth order, we give only the result for bcc lattices in terms of the numbers of equivalent graphs of various 
types involved, that is,

$$
\begin{aligned}
k_{B} T_{c}^{(4)} / J= & -4[l, l+\mu]+13[l, l+\mu+v]-3[l, l+\mu, l+v, l+\eta]-6[l, l+\mu+v, l+\eta]+3[l, l+\mu+v+\eta, l+\delta] \\
& -3[l, l+\mu+v+\eta=l+\delta]\} /\{2[l, l+\mu]-6[l, l+\mu, l+v]+3[l, l+\mu, l+v+\eta]\},
\end{aligned}
$$

in which we have introduced the symbols $[l, l+\mu, l+v, l+\eta],[l, l+\mu+v+\eta, l+\delta]$ and $[l, l+\mu+v$ $+\eta=l+\delta]$. These are numbers of equivalent graphs of the types illustrated in Figs. 3(a), 3(b), and 3(c), respectively.

From the above analysis, it is clear that only four distinct graphs are necessary in the calculation of $T_{c}$ up to the third order for fcc films. They are given in Figs. 1(a), 2(a), 2(b), and 2(c). On the other hand, we need six distinct graphs to calculate $T_{c}$ for bcc Ising films up to the fourth-order cumulant. These include the graphs as in Figs. 1(a), 2(a), 2(b), 3(a), 3(b), and 3(c).

\section{RESULTS AND DISCUSSION}

We are now in the position to embed graphs in the lattice. The procedure of calculation involves mainly the counting of equivalent graphs of every type for the lattice structure under consideration. As a simple illustration, we consider Fig. 1(a) for a bcc film of $L$ spin layers. At a given site $l$ in the bulk, there are eight nearest-

(a)

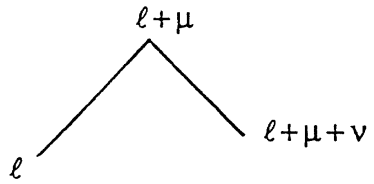

(b)

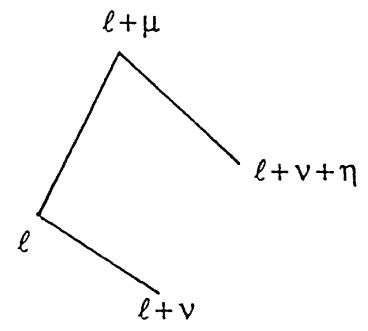

(c)

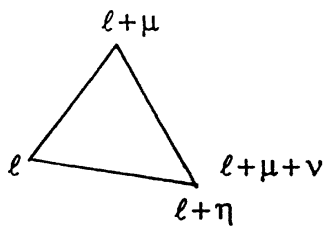

FIG. 2. Graphs contributing to $\left\langle S^{3}\right\rangle_{c}$ and topologically different from those in Fig. 1. neighboring sites. Therefore, $[l, l+\mu]=8(L-2)+8$ because every site on the two surfaces has only four nearest neighbors. Similarly, the number of the same graph is found to be $(12 L-8)$ for fcc films. The counting becomes quickly complicated and tedious as one goes to higher-order graphs, but it remains straightforward. Here we just give the final results. For bcc Ising films with $L$ spin layers, we find

$$
\begin{aligned}
& {[l, l+\mu]=8 L-8, \quad l \geq 2} \\
& {[l, l+\mu+v]=64 L-96, \quad L \geq 2} \\
& {[l, l+\mu, l+v, l+\eta]=512 L-896, \quad L \geq 2} \\
& {[l, l+\mu+v, l+\eta]=\left\{\begin{array}{l}
128, \quad L=2 \\
512 L-1024, \quad L \geq 3
\end{array}\right.}
\end{aligned}
$$

(a)

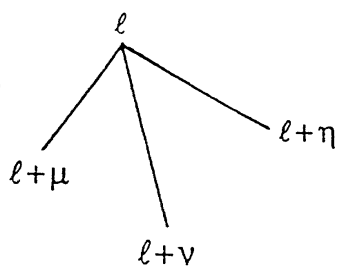

(b)

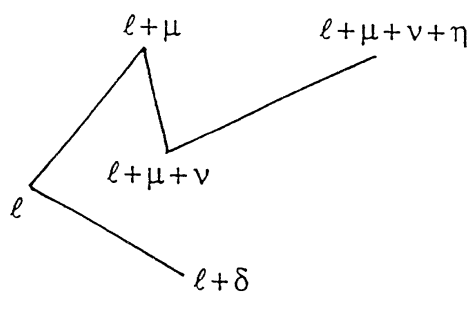

(c)

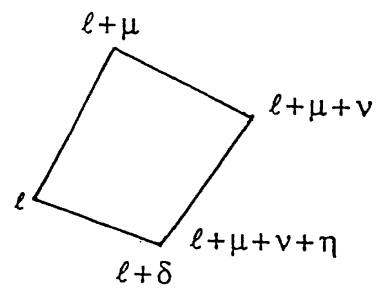

FIG. 3. Graphs contributing to $\left\langle S^{4}\right\rangle_{c}$ and topologically different from those in Figs. 1 and 2. 


$$
\begin{aligned}
& {[l, l+\mu+v+\eta, l+\delta]=\left\{\begin{array}{l}
512, \quad L=2 \\
3072, \quad L=3 \\
4096 L-9728, \quad L \geq 4
\end{array}\right.} \\
& {[l, l+\mu+v+\eta=l+\delta]=216 L-360, \quad L \geq 4 .}
\end{aligned}
$$

For fcc Ising films of $L$ layers, the numbers are

$$
\begin{aligned}
& {[l, l+\mu]=12 L-8, \quad L \geq 1} \\
& {[l, l+\mu+v]= \begin{cases}16, & L=1 \\
144 L-160, & L \geq 2\end{cases} } \\
& {[l, l+\mu+v, l+\eta]= \begin{cases}64, \quad L=1 \\
1024, \quad L=2 \\
1728 L-2560, \quad L \geq 3\end{cases} } \\
& {[l, l+\mu+v=l+\eta]= \begin{cases}0, & L=1 \\
48 L-48, & L \geq 2 .\end{cases} }
\end{aligned}
$$

Substituting the above results into Eqs. (16) and (19) -(22), we obtain

$$
\begin{aligned}
& k_{B} T_{c}^{(1)} / J=\frac{8 L-8}{L}, \quad L \geq 2 \\
& k_{B} T_{c}^{(2)} / J=\frac{7 L-11}{L-1}, \quad L \geq 2 \\
& k_{B} T_{c}^{(3)} / J= \begin{cases}2.889, \quad L=2 \\
\frac{146 L-314}{21 L-33}, \quad L \geq 3\end{cases} \\
& k_{B} T_{c}^{(4)} / J= \begin{cases}2.654, & L=2 \\
3.838, & L=3 \\
\frac{979 L-2561}{146 L-314}, & L \geq 4\end{cases}
\end{aligned}
$$

for bcc structures and

$$
\begin{aligned}
& k_{B} T_{c}^{(1)} / J=\frac{12 L-8}{L}, \quad L \geq 1 \\
& k_{B} T_{c}^{(2)} / J=\left\{\begin{array}{l}
3, L=2 \\
\frac{33 L-38}{2 L-7}, \quad L \geq 2
\end{array}\right. \\
& k_{B} T_{c}^{(3)} / J= \begin{cases}2.889, \quad L=1 \\
6.524, \quad L=2 \\
\frac{1050 L-1648}{99 L-114}, L \geq 3\end{cases}
\end{aligned}
$$

for fcc structures. In Figs. 4 and 5, we plot the critical temperature to different orders of cumulant expansion as functions of the number of spin layers in the film for bcc and fcc, respectively. The corresponding $T_{c}$ for bulk systems are also indicated in each case for comparison. They are given by $k_{B} T_{c} / J=6.4055 \pm 0.0010$ for bcc and $9.8290 \pm 0.0005$ for fcc, according to Ref. [16]. It is observed that the convergence of our cumulant expansion appears to be rather fast. In fact, we expect that accurate

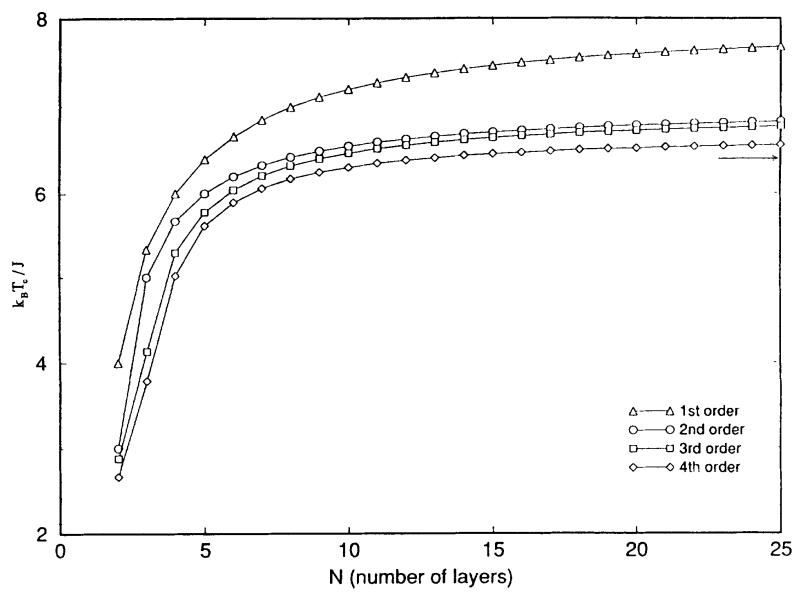

FIG. 4. Reduced critical temperature $\left(k_{B} T_{c} / J\right)$ calculated up to the fourth order as a function of $L$ for Ising films of bcc structure.

critical temperature can be obtained by including higherorder cumulants, provided that a computer code is developed to handle the counting of topologically equivalent graphs in every order.

We have demonstrated a powerful method that provides a straightforward and easy-to-handle approach to the calculation of the critical temperature of general Ising films of various lattice structures. Once the general expressions of cumulants such as those in Eqs. (14) and (15) are generated to the desired order, the critical temperature can be obtained for a given lattice structure to the desired accuracy simply by following the prescription of number counting of the distinct graphs. The procedure is easy to learn even for those who may not be familiar with the usual techniques of statistical mechanics.

The method should be particularly useful to help interpret the function $T_{c}(L)$ measured for Ising-like films which may exhibit structural changes as the film thick-

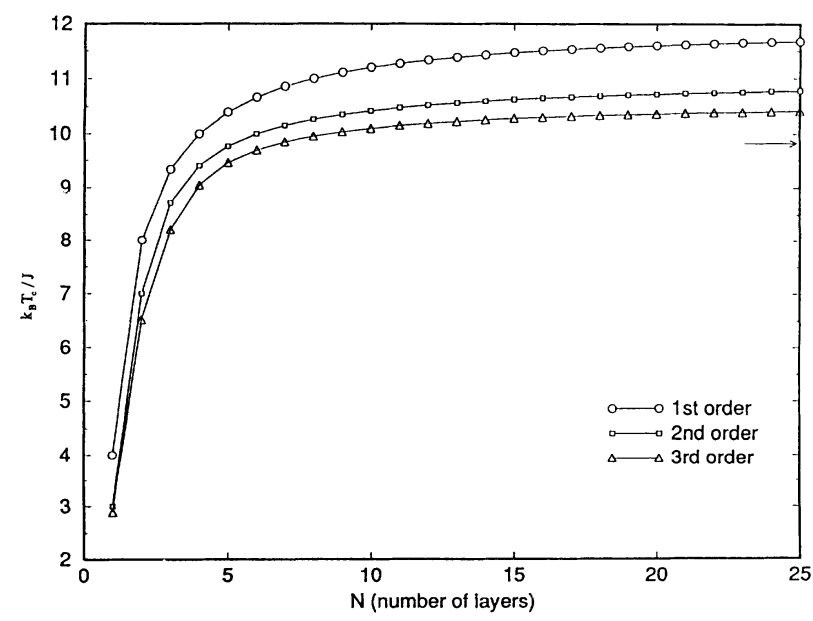

FIG. 5. Reduced critical temperature $\left(k_{B} T_{c} / J\right)$ calculated up to the third order as a function of $L$ for Ising films of fcc structure. 
ness increases during the growth [13]. Since our result is valid for arbitrary $L$, any structure change can readily be identified by comparing the curvature of the function $T_{c}(L)$ for different lattice structures with experimental data. We remark before concluding the paper that even if the external field is not considered in the present calculation, the surface anisotropy can still be taken into account in the theory to the extent that the order parameter remains unchanged throughout the lattice by allowing a variable coupling $J_{i j}$. This may complicate the calcula- tion somewhat, but will not introduce essential difficulty in the procedure.

\section{ACKNOWLEDGMENTS}

We would like to thank Francis M. Gasparini and John T. Ho for interesting discussions about the critical exponent. This research was supported in part by the New York State Institute on Superconductivity and in part by the National Science Foundation under Contract No. CHE-9196214.
[1] D. L. Lin, H. Che, and Y. Xia, Phys. Rev. A 46, 1805 (1992).

[2] X. Zheng, C.-I. Tan, and T. Chen, Phys. Rev. D 26, 2843 (1982).

[3] C.-I. Tan and X. Zheng, Phys. Rev. D 28, 3141 (1983).

[4] X. H. He, D. C. Xian, and Y. X. Song, Phys. Lett. B 153, 417 (1985); X. Zheng, T. Chen, and D. C. Xian, ibid. 154, 166 (1985).

[5] X. Zheng, Z. G. Tan, and D. C. Xian, Nucl. Phys. B 287, 171 (1987).

[6] H. Che, Y. Xia, H. M. Wang, and Y. X. Song, Commun. Theor. Phys. (Beijing) 12, 273 (1989).

[7] Y. Xia, H. Che, D. L. Lin, and H. M. Wang, Phys. Rev. B 46, 3125 (1992).

[8] T. W. Capeheart and M. E. Fisher, Phys. Rev. B 13, 5021
(1976).

[9] H. Strubbe, Comput. Phys. Commun. 8, 1 (1974).

[10] C. A. Ballentine, R. L. Fink, J. Araya-Pocket, and J. L. Erskine, Phys. Rev. B 412, 2631 (1990).

[11] M. Farle and K. Baberschke, Phys. Rev. Lett. 58, 511 (1987).

[12] Z. Q. Qiu, J. Pearson, and S. D. Bader, Phys. Rev. Lett. 67, 1646 (1991).

[13] Y. Li and K. Baberschke, Phys. Rev. Lett. 68, 1208 (1992).

[14] W. Durr, M. Taborelli, O. Paul, R. Germar, W. Gudat, D. Pescia, and M. Landolt, Phys. Rev. Lett. 62, 206 (1989).

[15] C. M. Schneider, P. Bressler, P. Schuster, and J. Kirschner, Phys. Rev. Lett. 64, 1059 (1990).

[16] M. F. Sykes, D. S. Gaunt, P. D. Roberts, and J. A. Wyles, J. Phys. A 5, 640 (1972). 